\title{
Gestação de alto risco: complicações decorrentes da tireoidite de Hashimoto
}

\author{
High-risk pregnancy: complications from Hashimoto's thyroiditis
}

Embarazo de alto riesgo: complicaciones de la tiroiditis de Hashimoto

Paula Xavier Lazarini ${ }^{1 *}$, Marília Medeiros de Matos $^{2}$, Milena Rodriguez Vallejos Vieira ${ }^{3}$, Milena Martins Magnus ${ }^{4}$, Marcella Almeida Fraga ${ }^{5}$, Maria Clara Bravieira Oliveira ${ }^{4}$, Jacob Henrique da Silva Klippel $^{6}$, Ana Flávia Gomes Viana ${ }^{1}$, Ana Carolina Grande ${ }^{7}$, Maria Luísa Barros Quintão Couto Parreira ${ }^{3}$.

\section{RESUMO}

Objetivo: Revisar e evidenciar as complicações da Tireoide de Hashimoto durante a gestação ao abordar repercussões para o feto e para a gestante; fisiopatologia, diagnóstico e tratamento da doença. Revisão bibliográfica: A tireoidite de Hashimoto é uma doença crônica e autoimune associada à hipofunção da glândula, caracterizada pela produção de autoanticorpos tireoidianos. É diagnosticada através da análise laboratorial, sintomatológica e histopatológica e tratada através de mudanças dos hábitos alimentares. $\mathrm{O}$ tratamento farmacológico é sustentado pelo uso de levotiroxina (L-T4). Em gestantes portadoras da doença, foram evidenciados riscos aumentados da pré-eclâmpsia, descolamento prematuro da placenta, anemia, hemorragia pós-parto, prematuridade, dentre outros. Além disso, em relação ao neonato, observa-se um maior risco de síndrome do desconforto respiratório, baixo peso ao nascer e retardo de crescimento, dentre outras repercussões ao longo da vida. Considerações finais: A fim de minimizar a morbimortalidade, fazemse necessárias mais pesquisas que relacionem a anatomia e a disfunção da glândula com o prognóstico da Tireoidite de Hashimoto, principalmente em gestantes.

Palavras-chave: Doença de Hashimoto, Gravidez de alto risco, Tireoidite autoimune, Hipotireoidismo.

\begin{abstract}
Objective: Review and Highlight the complications of Hashimoto's Thyroid during pregnancy, by addressing the repercussions for the fetus and the pregnant woman; pathophysiology, diagnosis and treatment of the disease. Literature review: Hashimoto's thyroiditis is chronic and autoimmune, associated with gland hypofunction, characterized by the production of thyroid autoantibodies. It is diagnosed through a laboratory, symptomatological and histopathological analysis and treated through changes in eating habits. Pharmacological treatment is supported by the use of levothyroxine (L-T4). In pregnant women with the disease, increased risks of pre-eclampsia, premature displacement of the placenta, anemia, postpartum hemorrhage, prematurity, among other things. In addition, in relation to the neonate, there is a greater risk of respiratory distress syndrome, low birth weight and growth retardation, among other repercussions throughout life. Final considerations: In order to minimize morbidity and mortality, further research is needed to correlate
\end{abstract}

\footnotetext{
${ }^{1}$ Pontifícia Universidade Católica de Minas Gerais (PUC-MG), Betim - MG.

* E-mail: profissionalpaulalazarini@gmail.com

2 Centro Universitário Unifacisa (UNIFACISA), Campina Grande - PB.

${ }^{3}$ Faculdade de Medicina do Vale do Aço (UNIVAÇO), Ipatinga - MG.

${ }^{4}$ Universidade José do Rosário Vellano (UNIFENAS), Belo Horizonte - MG.

5 Faculdades Unidas do Norte de Minas (FUNORTE), Montes Claros - MG.

6 Universidade Federal do Espírito Santos (UFES), Vitória - ES.

${ }^{7}$ Faculdade Assis Gurgacz (FAG), Cascavel - PR.
} 
the anatomy and dysfunction of the gland with the prognosis of Hashimoto's thyroiditis, especially in pregnant women.

Key words: Hashimoto disease, Pregnancy high-risk, Thyroiditis autoimmune, Hypothyroidism.

\section{RESUMEN}

Objetivo: Revisar y resaltar complicaciones de la tiroides de Hashimoto durante el embarazo, abordando repercusiones para el feto y la embarazada; fisiopatología, diagnóstico y tratamiento de la enfermedad. Revisión bibliográfica: La tiroiditis de Hashimoto es una enfermedad tiroidea autoinmune y crónica, asociada con hipofunción de la glándula, caracterizada por producción de autoanticuerpos tiroideos. Se diagnostica mediante análisis de laboratorio, sintomatológico e histopatológico y se trata mediante cambios en los hábitos alimentarios. El tratamiento farmacológico está respaldado por el uso de levotiroxina (L-T4). En mujeres embarazadas con la enfermedad, se evidenciaron mayores riesgos de preeclampsia, desplazamiento prematuro de la placenta, anemia, hemorragia posparto, prematurez, entre otros. Además, en relación al neonato, existe un mayor riesgo de síndrome de dificultad respiratoria, bajo peso al nacer y retraso del crecimiento, entre otras repercusiones a lo largo de la vida. Consideraciones finales: Para minimizar la morbilidad y la mortalidad, se necesitan más investigaciones para correlacionar la anatomía y disfunción de la glándula con el pronóstico de la tiroiditis de Hashimoto, especialmente en mujeres embarazadas.

Palabras clave: Enfermedad de Hashimoto, Embarazo de alto riesgo, Tiroiditis autoinmune, Hipotiroidismo.

\section{INTRODUÇÃO}

O período gestacional é marcado por mudanças fisiológicas, dentre elas na glândula tireoide materna, isso porque no primeiro trimestre ocorre um aumento de T4 livre pela supressão do hormônio tireoestimulante (TSH) (RANZCOG, 2018). Durante as alterações nesse mecanismo, pode haver hipotireoidismo, sendo o subtipo mais comum a tireoidite de Hashimoto. Nesse contexto, a doença da tireóide é prevalente em $2,5 \%$ das mulheres em idade reprodutiva, sendo que há complicações de 0,1 a $0,3 \%$ das gestações (BRASIL, 2010; SEGURA SA, 2018).

A doença de Hashimoto é caracterizada por um ataque autoimune às células tireotróficas, causando inflamação e interferindo na sua capacidade de produzir hormônios. Isso acontece porque há uma destruição dos folículos tireoidianos que liberam coloides contendo os hormônios triiodotironina (T3) e tiroxina (T4) no corpo, em consequência, ocorre um quadro de hipertireoidismo temporário. Com o avançar da doença, cada vez menos folículos estarão disponíveis na tireoide e o paciente evolui para um quadro de hipotireoidismo. No primeiro trimestre da gestação, os níveis séricos de TSH diminuem em decorrência da atividade do hormônio beta HCG, já no segundo e terceiro trimestre haverá retorno aos níveis fisiológicos (SHOKRI S, 2020).

Desse modo, os distúrbios tireoidianos na gestação culminam em efeitos obstétricos e fetais. Em relação às consequências obstétricas, destacam-se o aborto, o descolamento prematuro de placenta, pré-eclâmpsia e modificações na transferência de iodo para a placenta. Já os acometimentos fetais se relacionam com o baixo peso ao nascer, retardo mental, prematuridade e morte perinatal (ALEMU A, 2016). Além disso, nas mulheres, o hipotireoidismo causa alterações do ciclo menstrual e interfere nos mecanismos ovulatórios (QUINTINO-MORO A, 2014).

O rastreio da dosagem de hormônios T3 e T4 não é protocolo de conduta durante o pré-natal. Logo, recomenda-se que o ginecologista obstetra solicite um teste da função tireoidiana em todas as gestantes, além do acompanhamento do recém-nascido a fim de rastrear deficiências cognitivas. Somado a isso, também é necessária uma maior literatura científica acerca das alterações tireoidianas durante o período gestacional com a finalidade de avaliar o volume da tireoide e as características do parênquima e do nódulo (ALEMU A, 2016; SHOKRI S, 2020). 
Nesse sentido e considerando a relevância do assunto, o presente estudo tem por objetivo a realização de uma revisão narrativa da literatura que visa evidenciar as complicações da tireoidite de Hashimoto durante a gestação.

\section{REVISÃO BIBLIOGRÁFICA}

A Tireoidite de Hashimoto (TH), também chamada de tireoidite linfocítica crônica ou autoimune (AITD), faz parte do espectro de doenças autoimunes crônicas da tireoide e está associado a vários graus de hipofunção da glândula, com a produção de autoanticorpos tireoidianos, como os mais comuns, os anticorpos antireceptor do hormônio estimulante da tireoide (TSHR), da tireoperoxidase (TPO-Ab) e da tireoglobulina (TgAb) (LIONTIRIS MI e MAZOKOPAKIS EE, 2017; FRÖHLICH E e WAHL R, 2017).

Os anticorpos anti-receptor do hormônio estimulante da tireoide (TSHR), se desenvolvem a partir de uma modificação da subunidade A extracelular de derramamento, sendo ela o autoantígeno que inicia e/ou direciona a resposta autoimune ao TSHR. Já os anticorpos anti-TPO-Ab, são desencadeados por mutações, são indutores de estresse oxidativo, evidenciado pelo potencial antioxidante diminuído, produtos de glicosilação avançada e metabólitos de oxigênio no sangue. No caso dos anticorpos tireoglobulina (Tg-Ab), seu desenvolvimento é explicado devido à liberação maciça de antígenos após a destruição dos tireócitos ou geração de novos epítopos por uma conformação alterada e mais imunogênica da molécula de antitireoglobulina com alto conteúdo de iodo. De forma geral, é possível observar que depende do sucesso do complexo desenvolvimento de seus hormônios, e que uma modificação pode provocar ineficiência da glândula (FRÖHLICH E e WAHL R, 2017).

Além desses anticorpos que acometem o equilíbrio do sistema imunológico, fatores genéticos e ambientais podem desempenhar importante papel na doença. Nesse sentido, os fatores genéticos são os mais significativos, incluindo os principais genes de histocompatibilidade (que codificam antígenos leucocitários humanos), proteínas que regulam o sistema imunológico e genes específicos da tireoide. Por outro lado, a influência de fatores ambientais provoca a superprodução do sistema imune para os antígenos da tireoide, que incluem, excesso ou deficiência de nutrientes, exposição a metais pesados, toxinas, incluindo desreguladores endócrinos, como bisfenóis, ftalatos, drogas e outros. (IHNATOWICZ P, et al., 2020).

\section{Repercussões para a gestante}

Logo na primeira semana de gestação, ocorre a produção de gonadotrofina coriônica humana (hCG) pela placenta, atingindo concentração máxima por volta da $10^{\text {a }}$ semana e estabilização por volta da $20^{a}$ semana gestacional. Essa substância tem ação antagonista com o receptor de TSH e, juntamente com o aumento dos níveis séricos da globilina ligante de tiroxina e da deiodinase tipo 3 , contribuem com 0 aumento da concentração de T4 materno nos primeiros três meses de gravidez. Além disso, a tireoide materna aumenta sua produção hormonal em aproximadamente $40 \%$ pelas demandas metabólicas do momento fisiológico (MEDICI M, et al.,2015). Ranzcog (2018), acrescenta que a tireoide é uma glândula essencial para a saúde, apresenta importância na gravidez e a escassez do hormônio tireoidiano, hipotireoidismo, traz danos ao indivíduo. Apresenta, ainda, que a principal causa de hipotireoidismo na Austrália e na Nova Zelândia é a tireoidite de Hashimoto.

Miriam C, et al. (2020) estudaram cerca de 4.000 mulheres diagnosticadas com TH e dividiram-nas entre portadoras somente de TH e associada a doenças endócrinas. Entre as mulheres com endocrinopatias associadas, cerca de $5.64 \%$ apresentaram perda recorrente de gestação, em contraste dos $2.1 \%$ das com somente TH, revelando não só as consequências em mães portadoras de HT como também a importância da triagem para possíveis endocrinopatias associadas. Potlukova E, et al. (2012), realizaram um estudo que incluiu 5223 mulheres grávidas entre 9 e 12 semanas gestacionais com o propósito de verificar se o aumento da idade aumenta o risco de desenvolvimento de doenças autoimunes da tireoide durante a gravidez. Foi identificado que o aumento da idade, 30 anos ou mais, aumenta significativamente a eficácia do sistema de rastreamento de casos, entretanto, foi concluído que a idade da gestante não aumenta o risco de desenvolvimento de tireoidopatias autoimunes. 
Com o objetivo de avaliar o desenvolvimento de tireoidite pós-parto em mães com hipotireoidismo prévio, Moleti M, et al. (2020), estudaram 167 mulheres previamente diagnosticadas com TH e em tratamento com levotiroxina e dividiram-nas em um grupo eutireoidiano e outro hipotireoidiano. Ambos os grupos foram acompanhados desde o período preconcepção até um ano após o parto. Em sua conclusão, relataram um risco quatro vezes maior de desenvolver tireoidite pós-parto em mulheres que mantiveram o estado eutireoidiano durante o primeiro trimestre gestacional, sugerindo uma possível correlação entre o risco aumentado e a quantidade de tecido tireoidiano não afetado.

Mesmo o hipotireoidismo materno tendo inúmeras consequências tanto para a mãe quanto para o feto, a testagem sanguínea para o seu diagnóstico ainda é limitada (BLATT AJ, et al., 2012). Para isso, Blatt AJ, et al. (2012) obtiveram análises periódicas laboratoriais de anticorpo tireoperoxidase (TPO-Ab) e T4 livre de 502.036 mulheres grávidas com idade gestacional conhecida com a finalidade de analisar a taxa de testagem e de positividade para o hipotireoidismo durante a gestação. Em sua conclusão, reiteraram a importância da testagem hormonal tireoidiana durante a gestação e verificaram uma baixa taxa de acompanhamento pósparto.

\section{Repercussões fetais}

Durante os primeiros três meses de vida intrauterina, o embrião tem somente hormônios tireoidianos provenientes da mãe e, por causa dessa dependência, qualquer alteração nos níveis séricos da mãe pode influenciar no desenvolvimento embriológico. Baixos níveis séricos de hormônios tireoidianos afetam tanto a mãe quanto o filho, incluindo efeitos negativos neurológicos, comportamentais e cognitivos (MARAKA S, et al., 2016; SPRINGER D, et al., 2017).

Ao analisar o histórico das consequências fetais, houve uma mudança na principal causa de hipotireoidismo materno (antigamente era prevalente a deficiência de iodo e atualmente, a TH) e, com isso, por mais que fenotipicamente semelhantes, as alterações fisiopatológicas são distintas (ALEXANDER EK, et al., 2017).

Korevaar TIM, et al. (2016), por exemplo, analisaram aproximadamente 4.000 mães com baixos níveis de hormônios tireoidianos durante a gravidez e constataram uma significativa redução nos escores de QI, redução da massa cinzenta e do córtex em seus filhos seis anos após seus nascimentos. Também, Ghassabian A, et al. (2014) analisaram um total de 3727 de pares mãe e filho com os dados pré natais da função tireoidiana materna com o objetivo de analisar a relação entre hipotireoidismo materno e o QI dos filhos seis anos após o nascimento. Ao mesmo tempo, foi analisada também a relação dos volumes cerebrais globais, a espessura cortical e a área de superfície do cérebro entre os filhos de mães com baixa função tireoidiana e de mães eutireoidianas. Foi observado uma redução de 4,3 pontos no QI dos filhos das mães portadoras de hipotireoidismo em contraste com o outro grupo, mas nenhuma diferença nos parâmetros morfológicos cerebrais entre os dois grupos.

A duração e a gravidade do hipotireoidismo materno devem ser levadas em consideração quando analisadas as possíveis consequências fetais (ALEXANDER EK, et al., 2017). Entre elas, destacam-se um maior risco de defeitos perinatais e perda gestacional, parto prematuro, síndrome do desconforto respiratório neonatal, baixo peso ao nascer, retardo de crescimento, falha no desenvolvimento sexual, surdez e menor desenvolvimento motor e intelectual. Durante principalmente os primeiros meses de vida há prejuízo da diferenciação neuronal, hipodesenvolvimento do sistema nervoso central atrelado a um atraso na evolução cognitiva e motora, consequente redução do QI e nos níveis de atenção. A relação entre a TH e o desenvolvimento neurocognitivo ainda é muito incerta e são necessários mais estudos para compreendê-los (ALEXANDER EK, et al., 2017; SPRINGER D, et al., 2017).

Sem o tratamento adequado, as consequências são exponencialmente maiores, incluindo maior risco para retardo mental, aborto espontâneo, prematuridade e crescimento intrauterino ainda mais restrito (BRASIL, 2010). Entretanto, Lazarus JH, et al. (2012), recrutaram aproximadamente 22.000 mulheres portadoras de hipotireoidismo com gestações de até 15 semanas e seis dias e dividiram-nas em um grupo controle e um grupo de triagem. A ideia do estudo era analisar a influência que a triagem pré-natal e o 
tratamento materno com levotiroxina diária na melhora da função cognitiva das crianças três anos após o parto. Contudo, não foram encontradas melhoras significativas nestas crianças em comparação com as nascidas de mães que não realizaram o tratamento.

\section{Diagnóstico}

Como dito anteriormente, no período da gestação ocorrem mudanças fisiológicas na função tireoidiana materna sendo necessária uma análise laboratorial, sintomatológica e às vezes até mesmo histopatológica criteriosa para diagnosticar essa disfunção, em razão de prováveis complicações obstétricas caso não tratada, atentando-se a possíveis diagnósticos diferenciais (ALMEIDA, et al., 2015).

Inicialmente, para diagnóstico do hipotireoidismo materno, é necessária uma análise laboratorial do hormônio tireoestimulante (TSH) sérico, que é o indicador mais sensível para confirmação e o principal hormônio avaliado para diferentes patologias tireoidianas, o qual encontra-se elevado no hipotireoidismo primário. $\mathrm{Na}$ TH é importante a dosagem do anticorpo peroxidase tireoidiano (TPO-Ab), que está presente em cerca de $95 \%$ das gestantes. Além de ser um importante marcador dessa etiologia, é um marcador de risco de pacientes que podem desenvolver o hipotireoidismo durante a gestação ou pós-parto. Para o diagnóstico de doença tireoidiana autoimune também se faz necessário a pesquisa de anticorpos tireoglobulina ( $\mathrm{Tg}-\mathrm{Ab}$ ) presentes em cerca de $60 \%$ das gestantes, sendo menos específico para a tireoidite de Hashimoto, podendo ser um indicador de uma expressão imune inicial, diferente do TPO-Ab que podem ser uma resposta imune posterior e crônica. Já a análise do T4 livre deve ser criteriosa, pois o mesmo pode sofrer variações de acordo com cada trimestre da gestação (FRÖHLICH E e WAHL R, 2017).

A sintomatologia do hipotireoidismo de Hashimoto na gestação é baseada em sintomas sistêmicos e locais, sendo uma análise auxiliar para diagnóstico. Já a análise histopatológica não é realizada rotineiramente, por ser um procedimento invasivo, sendo necessária para diagnósticos diferenciais, como em tumores tireoidianos (ALEXANDER EK, et al., 2017).

\section{Tratamento}

Qualquer gestante diagnosticada com hipotireoidismo deve receber cuidados adequados. Sendo a tireoidite de Hashimoto $(\mathrm{TH})$ a causa mais comum dessa doença em populações sem deficiência de iodo, a intervenção farmacológica é feita com levotiroxina (L-T4), que deve ser administrada uma vez ao dia em jejum. A fim de manter o nível manter o hormônio tireoestimulante (TSH) na faixa fisiológica, de acordo com cada trimestre de gestação, a administração oral do hormônio sintético L-T4 segue a dosagem de 1,6 a 1,8 microgramas por quilograma (ALMEIDA JP, et al., 2015).

$\mathrm{O}$ tratamento do quadro de $\mathrm{TH}$ também consiste em mudanças de hábitos alimentares. $\mathrm{O}$ iodo, por exemplo, é essencial para a função tireoidiana adequada. Sua ingestão diária recomendada para mulheres grávidas ou lactantes é de $250 \mu \mathrm{g}$, sendo atingida por meio de frutos do mar, iogurte, leite de vaca, ovos e algumas frutas. Contudo, muitos países já suprem o problema da deficiência desse micronutriente com a adição de iodo no sal. Logo, tendo em vista os indícios de que a alta ingesta de iodo está associada à maior ocorrência de hipertireoidismo, a suplementação de iodo deve ser desencorajada, pois, além de não ser benéfica, é também possivelmente prejudicial. Outros minerais, como o selênio (Se), parecem ajudar na resposta imune da tireoide, uma vez que esse é o órgão do corpo humano que mais armazena o elemento. Dessa forma, a suplementação de selênio pode intensificar a ação da glutationa peroxidase e da tioredoxina redutase plasmática, além de atenuar as concentrações tóxicas de peróxido de hidrogênio e hidroperóxidos lipídicos, resultantes da síntese dos hormônios tireoidianos. O Se está presente no solo e entra na cadeia alimentar através das castanhas, sementes e cogumelos. Vê-se também uma associação entre deficiência de vitamina $D$ e a patogênese da TH. Assim, levando em consideração o baixo custo e os escassos efeitos colaterais, indica-se a adoção dessa vitamina na terapêutica (LIONTIRIS MI e MAZOKOPAKIS EE, 2017).

Além disso, observa-se, em pacientes com TH, a disbiose intestinal. A flutuação dos níveis de hormônio tireoidiano causada pela doença afeta a composição da microbiota. Por isso, para melhorar a qualidade da flora bacteriana, deve atentar-se ao tipo de gordura consumida, pois a ingestão abundante de ácidos graxos saturados é danosa. Ademais, opta-se pela escolha de produtos com o menor grau de processamento, como 
grãos inteiros, por conterem mais fibras alimentares. Quanto aos peixes gordurosos, recomenda-se o consumo duas vezes na semana. Entre 1 a 2 gramas de ômega-3 devem ser fornecidos na dieta diária, via alimentos, uma vez que são uma escolha melhor do que a suplementação na forma de cápsula, que só deve ser introduzida com acompanhamento médico (IHNATOWICZ P, et al., 2020).

\section{CONSIDERAÇÕES FINAIS}

A partir dos dados supracitados, é perceptível a influência dos distúrbios tireoidianos na gestação. Estes resultam em efeitos obstétricos e fetais, que podem ser minimizados, e até evitados, a partir da realização de teste da função tireoidiana em todas as gestantes e do acompanhamento do recém-nascido, com o intuito de rastrear deficiências cognitivas. É fundamental um maior número de pesquisas científicas relacionadas às alterações tireoidianas durante a gravidez. Isso para avançar nos estudos e avaliar tópicos ainda não consolidados acerca do tema, como: volume da tireoide, características do parênquima e do nódulo, e consequentemente, promover ainda mais recursos para uma gestação saudável em mulheres que possuem essa doença.

\section{REFERÊNCIAS}

1. ALEMU A, et al. Thyroid hormone dysfunction during pregnancy: A review. International Journal of Reproductive Biomedicine: 2016; 14(11): 677-686.

2. ALEXANDER EK, et al. 2017 Guidelines of the American Thyroid Associaon for the Diagnosis and Management of Thyroid Disease During Pregnancy and the Postpartum. Thyroid: 2017; 27: 315-389.

3. ALMEIDA JP, et al. Hiporeoidismo e gestação: diagnóstico e conduta. Revista Hupe, 2015; 14(2): 54-58.

4. BLATT AJ, et al. National status of testing for hypothyroidism during pregnancy and postpartum. J Clin Endocrinol Metab, 2012; 97(3): 777-784.

5. BRASIL. Gestação de alto risco. Manual Técnico do Ministério da Saúde. 2010. Disponível em: gestacao_alto_risco.pdf (saude.gov.br). Acesso em: 02 de Abril de 2021.

6. FRÖHLICH E, WAHL R. Thyroid autoimmunity: role of anti-thyroid antibodies in thyroid and extra-thyroidal diseases. Frontiers in immunology, 2017; 8: 521.

7. GHASSABIAN A, et al. Downstream Effects of Maternal Hypothyroxinemia in Early Pregnancy: Nonverbal IQ and Brain Morphology in School-Age Children. J Clin Endocrinol Metab, 2014; 99(7): 2383-2390.

8. IHNATOWICZ P, et al. The importance of nutritional factors and dietary management of Hashimoto's thyroiditis. Annals of Agricultural and Environmental Medicine: AAEM, 2019; 27: 184-193.

9. KOREVAAR TIM, et al. Association of maternal thyroid function during early pregnancy with offspring IQ and brain morphology in childhood: a population-based prospective cohort study. Lancet Diabetes Endocrinol, 2016; 4: 35-43.

10. LAZARUS JH, et al. Antenatal Screening for Hypothyroidism and Childhood Cognitive Impairment. N Engl J Med, 2012; 366: 493-501.

11. LIONTIRIS M, MAZOKOPAKIS E. A concise review of Hashimoto thyroiditis (HT) and the importance of iodine, selenium, vitamin $D$ and gluten on the autoimmunity and dietary management of HT patients. Points that need more investigation. Hellenic Journal of Nuclear Medicine, 2017; 20(1): 51-56.

12. MARAKA S, et al. Subclinical Hypothyroidism in Pregnancy: A Systematic Review and Meta-Analysis. Thyroid, 2016; 26: 580-590.

13. MEDICI M, et al. Thyroid Function in Pregnancy: What is Normal?. Clin Chem, 2015; 61: 704-13.

14. MIRIAM C, et al. Recurrent Pregnancy Loss in Women with Hashimoto's Thyroiditis with Concurrent Non-Endocrine Autoimmune Disorders. Thyroid, 2020; 30: 457-462.

15. MOLETI M, et al. Postpartum Thyroiditis in Women With Euthyroid and Hypothyroid Hashimoto's Thyroiditis Antedating Pregnancy. The Journal of clinical endocrinology and metabolism, 2020; 105(7): dgaa197.

16. POTLUKOVA E, et al. Is age a risk factor for hypothyroidism in pregnancy? An analysis of 5223 pregnant women. J Clin Endocrinol Metab, 2012; 97(6): 1945-1952.

17. QUINTINO-MORO A, et al. High prevalence of infertility among women with graves' disease and hashimoto's thyroiditis. Int J Endocrinol, 2014; 2014 : 982705.

18. RANZCOG. Subclinical hypothyroidism and hypothyroidism in pregnancy. The Royal Australian and New Zealand College of Obstetricians and Gynaecologists, 2018; 2018: 1-11.

19. SHOKRI S, et al. Thyroid volume and nodular and diffuse thyroid diseases by ultrasonography in pregnant women: $A$ case-control study. J Res Med Sci, 2020; 25: 13.

20. SPRINGER D, et al. Thyroid in pregnancy: From physiology to screening. Crit Rev Clin Lab Sci, 2017; 54: $102-116$.

21. SEGURA SA, et al. Recomendaciones para el diagnóstico y seguimiento del feto y del recién nacido hijo de madre con patología tiroidea autoinmune. Anales de Pediatría, 2018; 89: 254.e1-254.e7. 